\title{
Article \\ Stiffness Modelling and Performance Evaluation of a Soft Cardiac Fixator Flexible Arm with Granular Jamming
}

\author{
Likun Gao ${ }^{1,2}$, Yanlin He ${ }^{1,2, *}$, Hangwei Zhu ${ }^{1,2}$, Guangkai Sun ${ }^{1,2}$ and Lianqing Zhu ${ }^{1,2}$ \\ 1 Key Laboratory of the Ministry of Education for Optoelectronic Measurement Technology and Instrument, \\ Beijing Information Science \& Technology University, Beijing 100192, China; gaolikun0@163.com (L.G.); \\ hangweizhu@126.com (H.Z.); guangkai.sun@bistu.edu.cn (G.S.); zlq_2018@sina.com (L.Z.) \\ 2 Beijing Laboratory of Optical Fiber Sensing and System, Beijing Information Science \& Technology University, \\ Beijing 100016, China \\ * Correspondence: heyanlin@bistu.edu.cn
}

check for updates

Citation: Gao, L.; He, Y.; Zhu, H.; Sun, G.; Zhu, L. Stiffness Modelling and Performance Evaluation of a Soft Cardiac Fixator Flexible Arm with Granular Jamming. Machines 2021, 9, 303. https://doi.org/10.3390/ machines 9120303

Academic Editor: Edoardo Sinibaldi

Received: 28 October 2021

Accepted: 19 November 2021

Published: 23 November 2021

Publisher's Note: MDPI stays neutral with regard to jurisdictional claims in published maps and institutional affiliations.

Copyright: (c) 2021 by the authors. Licensee MDPI, Basel, Switzerland. This article is an open access article distributed under the terms and conditions of the Creative Commons Attribution (CC BY) license (https:// creativecommons.org/licenses/by/ $4.0 /)$.

\begin{abstract}
To meet the practical application requirements of cardiac fixation during off-pump coronary artery bypass surgery, a soft cardiac fixator with a flexible arm was previously designed. To enable the soft cardiac fixator to adapt to uncertain external forces, this study evaluates the variable-stiffness performance of the flexible arm. First, the flexible arm was simplified as a soft silicone manipulator measuring $60 \mathrm{~mm} \times 90 \mathrm{~mm} \times 120 \mathrm{~mm}$, which can actuate, soften, or stiffen independently along the length of the arm by combining granular jamming with input pressure. Then, the soft manipulator was modelled as a cantilever beam to analyse its variable-stiffness performance with granular jamming. Next, based on theoretical analysis and calculations, many experiments were conducted to evaluate the variable-stiffness performance of the soft manipulator. The experimental results demonstrated that the variable-stiffness performance is influenced by the flexible arm length, the size of the granules, and the input pressure.
\end{abstract}

Keywords: soft surgical robot; cardiac fixation; flexible arm; variable stiffness; granular jamming

\section{Introduction}

During off-pump coronary artery bypass (OPCAB) surgery, the beating of the heart increases the difficulty of surgery, leading to longer surgical times and unfavourable patient outcomes [1]; thus, cardiac fixators are an indispensable surgical tool. However, cardiac fixators currently used in surgery are rigid and lack variable stiffness; therefore, when used to manipulate and fully invert the heart, they can lead to a loss of systemic pressure and decreased cardiac performance [1].

In recent years, significant progress has been made in the field of flexible robotics for surgical applications because they offer unique opportunities in areas where conventional rigid robots are not a viable solution [2-6]. Flexible robots are robots that exhibit stretchable material characteristics, as opposed to the rigid characteristics of conventional robots, and because of these stretchable material characteristics, they exhibit large-scale deformation and better adaptability to complex human organs and tissues $[7,8]$. Flexible robots that mimic the movement of animals have also been designed, e.g., flexible cylindrical manipulators, flexible grippers, starfish, and octopus tentacles. Moreover, their applications in bending locomotion and object grabbing have been demonstrated in previous studies [9-12]. For example, Ranzani et al. [5] analysed the advantages of flexible robotic technology for minimally invasive surgery, noting that a flexible robot can bend and extend flexibly through narrow areas to the distal end of the surgical operation. Moreover, soft surgical robots can adapt to various complex tissues, owing to their unlimited degrees of freedom (DoF), flexibility, and adaptability. Sandesh et al. [13] developed a five-finger manipulator that uses flexible sensors to control the robot hand. This manipulator uses multiple joints to control the degrees of freedom and complete functions such as grasping 
objects. Furthermore, Ranzani et al. [14] presented a concept design of a modular flexible manipulator for minimally invasive surgery and discussed its possible applications in the surgical field. This manipulator comprised a series of modules, each containing a silicone matrix with pneumatic chambers for three-dimensional (3D) motion, and one central channel for integrating a granular-jamming-based stiffening mechanism. Zouari et al. [15] proposed a brushless DC motor-driven flexible joint manipulator (BDCM), which improves the accuracy of the flexible manipulator drive control by compensating for all of the uncertainties and disturbances of the flexible joint manipulator. Payne et al. [16] proposed an implantable flexible robotic device to augment cardiac function during isolated left or right heart failure by applying rhythmic loading to either ventricle. Recovery of blood flow and pressure from the baseline heart failure condition was demonstrated, as well as significant reductions in diastolic ventricle pressure, which ensures a sustainable cardiac output and is crucial for the treatment of heart failure. Although many such flexible manipulators are available for surgical applications, most of these robots lack variable stiffness.

Variable-stiffness surgical robots that are similar to the locking mechanisms of endoscopes have previously been proposed [17-19]. The locking mechanisms utilise phasechange materials, such as wax or solder, to realise the transition of surgical robots between the liquid and solid states. However, these systems only freeze the shape of the instrument during surgery and do not allow active interaction between the body of the instrument and the surroundings. Instead, a variable-stiffness device should enable adjustment of this interaction and of the force exerted against the surroundings. For example, through the pulling or slackening of wires, tendon-driven robots can achieve variable stiffness [20]. Khodambashi et al. proposed heterogeneous hydrogel structures with programmable spatiotemporal deformation using addressable and tunable hydrogel building blocks [21]. Park et al. proposed magnetic deformation of triangular and quadrilateral micropillars and real-time manipulation of droplet shape by bending and twisting an array of micropillars with non-circular cross-sections driven [22]. Moreover, some snake-like robots use micro-motors to achieve variable stiffness; however, the stiffness force is insufficient for surgical operations $[23,24]$. Jiang presented a variable-stiffness sheath based on shape memory alloy (SMA) for the continuum manipulators. By adjusting the voltage across the sheath, the rigidity of the sheath can be changed with the phase change of SMA between austenite and martensite [25]. Dupont designed a manipulator that uses curved tubes to vary its stiffness; however, the stiffness close to the tip is lower than that at the end of the manipulator [26]. Suresh et al. designed a flexible manipulator composed of an asymmetric flexible pneumatic actuator (AFPA). The shape of the flexible manipulator arm is changed by pneumatic control and the stiffness of the top of the manipulator arm increases with the eccentricity [27]. Cha proposed a three-DoF flexible finger mechanism using a spring as the backbone, and derived the stiffness model of the flexible finger using the linear spring model and transformed it into a torsion spring model [28]. Stilli proposed a controllable solution for the stiffness of robot joints based on an air-pressurized chamber. When inflated to a high pressure, the mesh silicone cavity is like a rigid link; the air pressure is reduced and the connecting rod is softened [29]. Morrison presented a general 3D model of the link stiffness using the screw theory and compliance matrices, as well as a planar model for the lateral and torsional stiffnesses [30]. In addition, using the phase change properties of low melting point alloys to hydraulically drive and change the stiffness of the manipulator by heating and cooling can also be achieved [31,32]. The heated PET tube can also be converted between liquid and solid states of the substance by adjusting the temperature. However, since the present device is considered for medical and clinical applications, its adjustable temperature range is small [33]. In contrast, the state change achieved based on granular jamming theory is not affected by temperature and can be achieved by simply adjusting the air pressure. Some studies have investigated granular jamming, which serves as an actuator and universal joint [17,34]. John designed a universal gripper, consisting of a mass of granular material encased in an elastic membrane. The gripper realizes the object gripping by being vacuum hardened, later utilizing positive pressure to reverse this 
transition, thereby releasing the object and returning to a deformable state [35]. In these studies, when an external force was applied to the granular system, its stiffness changed arbitrarily according to different shapes of the granular system. For example, the viscosity of a mechanical element with variable stiffness produced by vacuum pressure was shown to vary with element transformation, which was controlled by air pressure [34]. Additionally, in a manipulator that implements variable stiffness through granular jamming, the grains changed from solid-like to liquid-like states because of the input pressure, resulting in more force than conventional manipulators [17].

The novelty of this study lies in designing a biologically inspired cardiac fixator with a flexible arm that can achieve variable stiffness using granular jamming by considering the design criteria of the fixator (e.g., size of the tube, required forces). Almost no research has previously been conducted on flexible cardiac fixators capable of achieving variable stiffness during OPCAB surgery. The hardening of the flexible arm is achieved by pumping negative pressure, and the stiffness of the flexible arm is adjusted by adjusting the amount of negative pressure. First, the flexible arm was simplified as a soft manipulator and modelled as a cantilever beam to evaluate its variable-stiffness performance. Second, a soft silicone manipulator measuring $60 \mathrm{~mm} \times 90 \mathrm{~mm} \times 120 \mathrm{~mm}$ was fabricated, and its variable-stiffness performance was experimentally evaluated using different granule sizes and under different input pressures.

The remainder of this paper is organised as follows. Section 2 summarises the previously described flexible cardiac fixator with a flexible arm and a model of the flexible arm. Section 3 presents and discusses the experimental results of the soft silicone manipulator. Section 4 presents the conclusions and future research directions.

\section{Methods}

\subsection{Design of the Soft Cardiac Fixator with Variable Stiffness}

Considering the requirements of cardiac fixation during an OPCAB surgery, a biologically inspired soft cardiac fixator with a flexible arm, which could achieve variable stiffness through granular jamming and pneumatic control, was designed (Figure 1a). The structural design of the soft cardiac fixator is shown in Figure 1b. Two soft cardiac fixators are used simultaneously during the surgery. The soft cardiac fixator has a U-shaped structure with eight symmetrically bionic octopus suckers, which can be used for adsorption and fixation of the heart. When the sucker attaches to the surface of the heart, it adapts to that surface, thus creating a seal, and reduces its thickness by contracting the radial muscles, thereby increasing its attachment to the surface through pneumatic control. The flexible arm achieves variable stiffness through granular jamming with three pneumatic tubes. Additionally, it comprises a rigid tail structure with five vacuuming cavities and other connectors.

Granular jamming is a method used to realise stiffness transformation in soft manipulators. When the internal pressure of the soft manipulator increases, some granules inside the manipulator become squeezed together, enabling the manipulator to achieve variable stiffness. Conversely, when the internal pressure of the soft manipulator decreases, some granules are loosened, regaining the flexibility of the manipulator. For the soft cardiac fixator described in this study, the flexible supporting arm not only achieves variable stiffness through granular jamming with pneumatic control, but also allows flexible deformation and rigid support of the entire soft cardiac fixator; i.e., soft-hard conversion. The variable-stiffness performance of the flexible arm is also related to the arm length and the size of the granules.

\subsection{Modelling the Variable Stiffness of the Flexible Arm}

To analyse the granular jamming behaviour, the flexible arm was simplified into a soft manipulator, and then modelled as a fixed cantilever beam bearing a force at its tip; thus, the soft manipulator exhibits the normal and deflected states shown in Figure 2. In the deflected state, region 1 is under tension and the granules are separated from each 
other, region 2 undergoes compression, and region 3 contains granules in their normal state $[36,37]$.
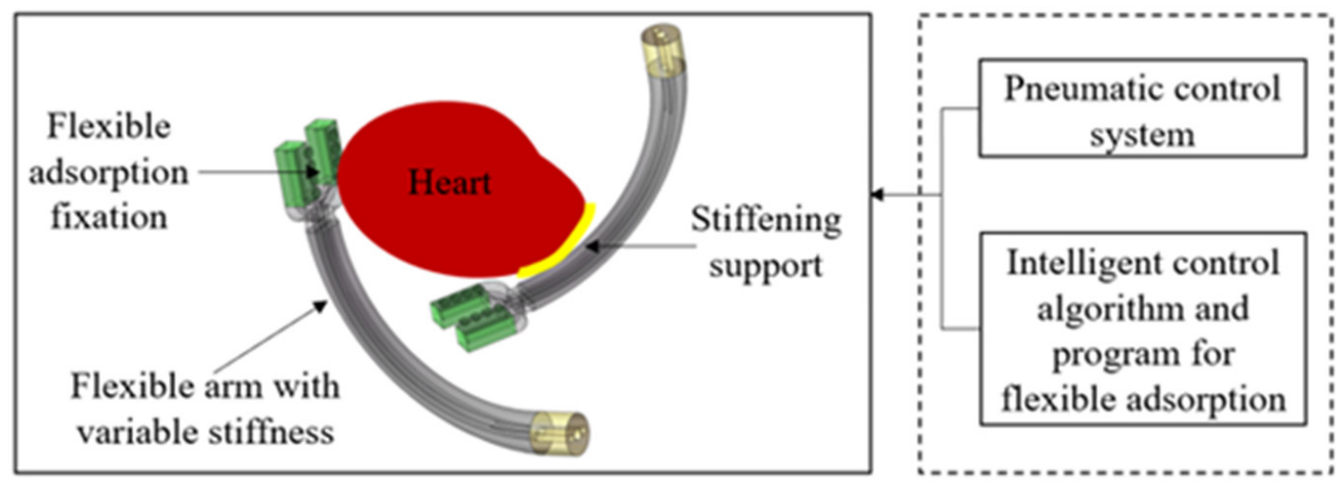

(a)

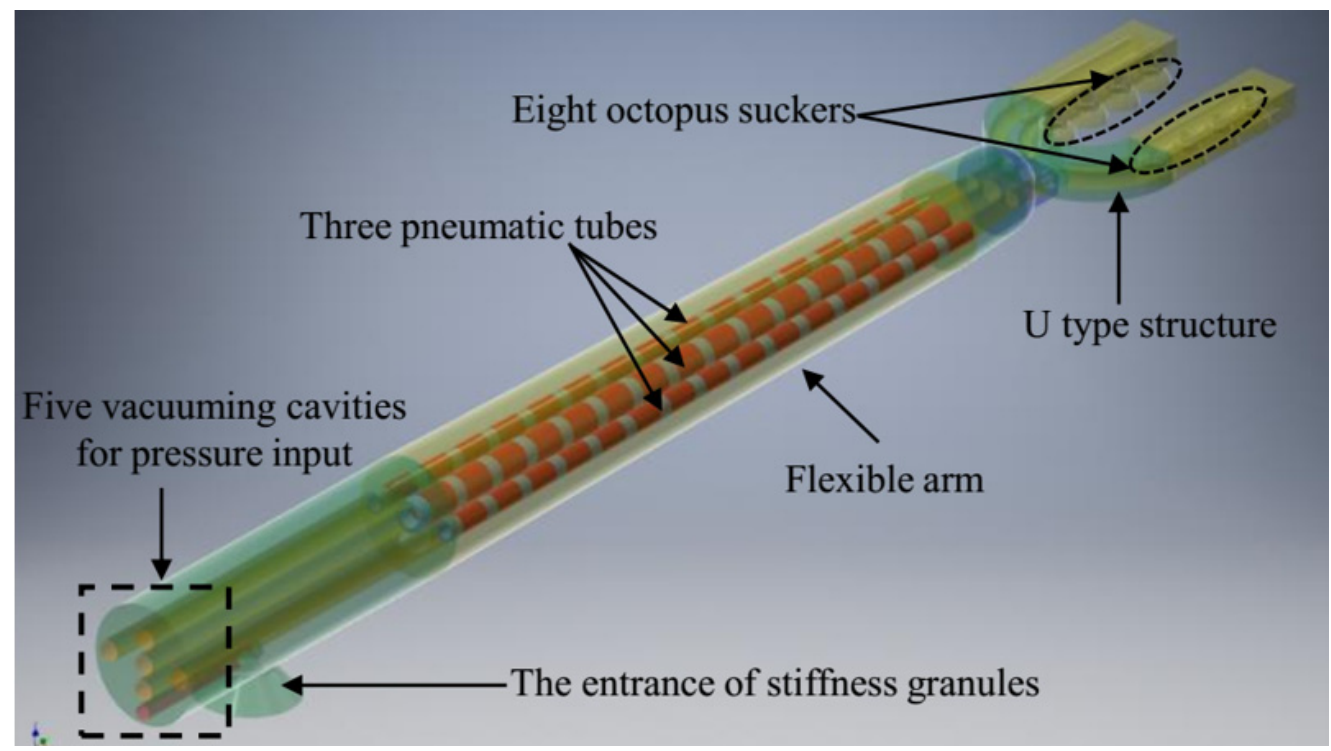

(b)

Figure 1. Schematic and structural design of the soft cardiac fixator with variable stiffness. (a) Schematic of the developed cardiac fixator with a flexible arm. (b) Structural design.

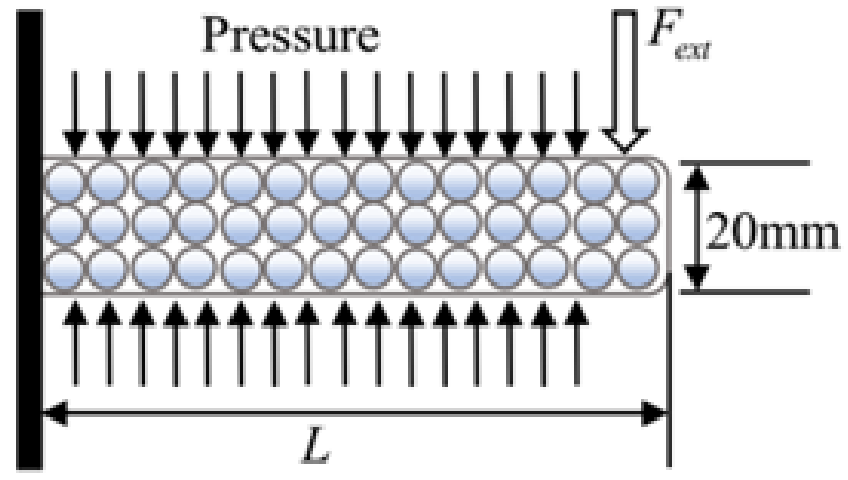

(a)

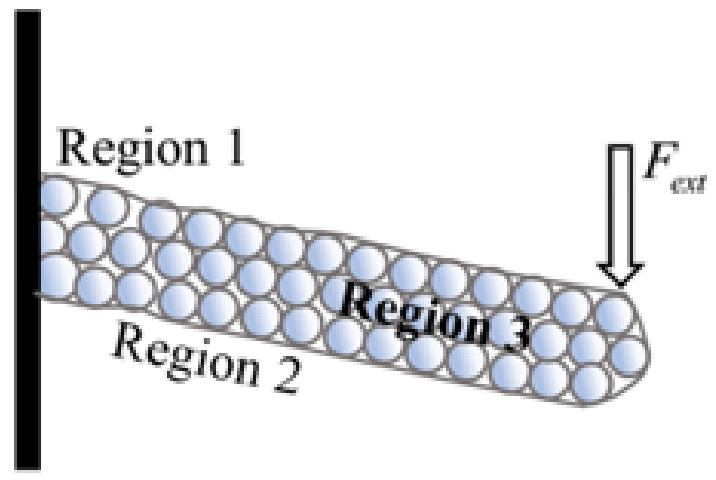

(b)

Figure 2. Modelling of the soft manipulator in different states. (a) Normal state. (b) Deflected state. 
The total bending moment of the soft manipulator can be expressed as:

$$
|M|=|L|\left|F_{\text {ext }}\right|
$$

where $M, L$, and $F_{\text {ext }}$ represent the total moment, length of the soft manipulator, and external force applied to the soft manipulator, respectively. The moment at a single point along the flexible arm can be expressed as:

$$
|M|=F_{\text {ext }}(L-d)
$$

where $d$ represents the length to the fixed end. When $d$ increases and becomes closer to the tip, the moment decreases linearly. Thus, when the fixed end bears the maximum moment, the flexible arm exhibits maximum bending at that point.

To calculate the bending of the flexible arm, it is considered that the flexible arm has a rectangular cross section, i.e.,

$$
y(d)=\frac{12 F_{e x t}}{E w t^{3}}\left(\frac{L d^{2}}{2}-\frac{d^{3}}{6}\right)
$$

where $w$ and $t$ represent the width and thickness of the flexible arm, respectively, and $y(d)$ represents the perpendicular displacement of the flexible arm along distance $d$.

\subsection{Experiments on the Soft Manipulator}

To evaluate the feasibility and variable-stiffness performance of the flexible arm of the soft cardiac fixator, a series of experiments was conducted. The flexible arm was simplified as a soft manipulator made from silicone material Ecoflex00-50 A and B. The experimental setup consisted of a soft manipulator, pneumatic control system, displacement platform, force measurement system, and a computer (Figure 3). The soft manipulator was fabricated by mixing equal amounts of Ecoflex00-50 A and B, and then pouring the mixture into moulds of different lengths. The pneumatic control system employed the FUJIWARA 750D vacuum pump to input pressure to the soft manipulator, which enabled direct measurement of the pressure value. The vacuum pump was connected to the soft manipulator through a filter; the connectors were designed with a honeycomb structure to prevent granules from being sucked into the vacuum pump. The KMS 40 Force/Torque sensor, used for force measurement of the soft manipulator, was mounted on the displacement platform. The movement of the sensor was controlled by the linear displacement platform. Software was used to control the platform position and log the pressure and force data.

Three groups of experiments were conducted to evaluate the performance of the soft manipulator. The first group of experiments was designed to evaluate the effect of granule size on the variable stiffness of the soft manipulator. For this group, three types of granule were packed into the soft manipulator, namely, millet, round rice, and red beans, having approximate diameters of 2, 4, and $6 \mathrm{~mm}$, respectively (Figure 4a). Experiments in this group were conducted with a fixed length of the soft manipulator and fixed pressure. The second group was designed to evaluate the effect of manipulator length on the variable stiffness - for this purpose, three manipulators of different lengths were used: 60, 90, and $120 \mathrm{~mm}$ (Figure 4b). Experiments in this group were conducted with a fixed input pressure and granule size. The third group was designed to evaluate the effect of pressure on the variable stiffness-for this purpose, input pressures of 44,56, 68, 80, and $92 \mathrm{kPa}$ were used. In Section 3, each group is discussed in detail and the related figures are presented.

In all experiments, the soft manipulator first touched the KMS 40 sensor (displacement platform movement $=0 \mathrm{~mm}$ ). Then, the displacement platform pushed the sensor leftward, with the displacement varying between 0 and $10 \mathrm{~mm}$, and holding for $5 \mathrm{~s}$. Each experiment was repeated four times.

To lucidly illustrate the experimental process, Figure 5 is presented. First, the manipulator length was fixed at $60 \mathrm{~mm}$ and millets (diameter: $2 \mathrm{~mm}$ ) were packed into it 
before a pressure of $80 \mathrm{kPa}$ was applied and maintained. Consequently, the manipulator touched the KMS 40 sensor, without displacement; then, the displacement platform pushed the sensor toward the manipulator, displacing it from $0 \mathrm{~mm}$ to $10 \mathrm{~mm}$ (Figure 5a). Subsequently, the same experiment was performed but with different granules; namely, round rice (diameter: $4 \mathrm{~mm}$ ) (Figure $5 \mathrm{~b}$ ) and red beans (diameter: $6 \mathrm{~mm}$ ) (Figure $5 \mathrm{c}$ ). With the displacement platform holding for $5 \mathrm{~s}$ at each displacement interval, each experiment was repeated 10 times and the final result of the force is the average of those from the 10 experiments.

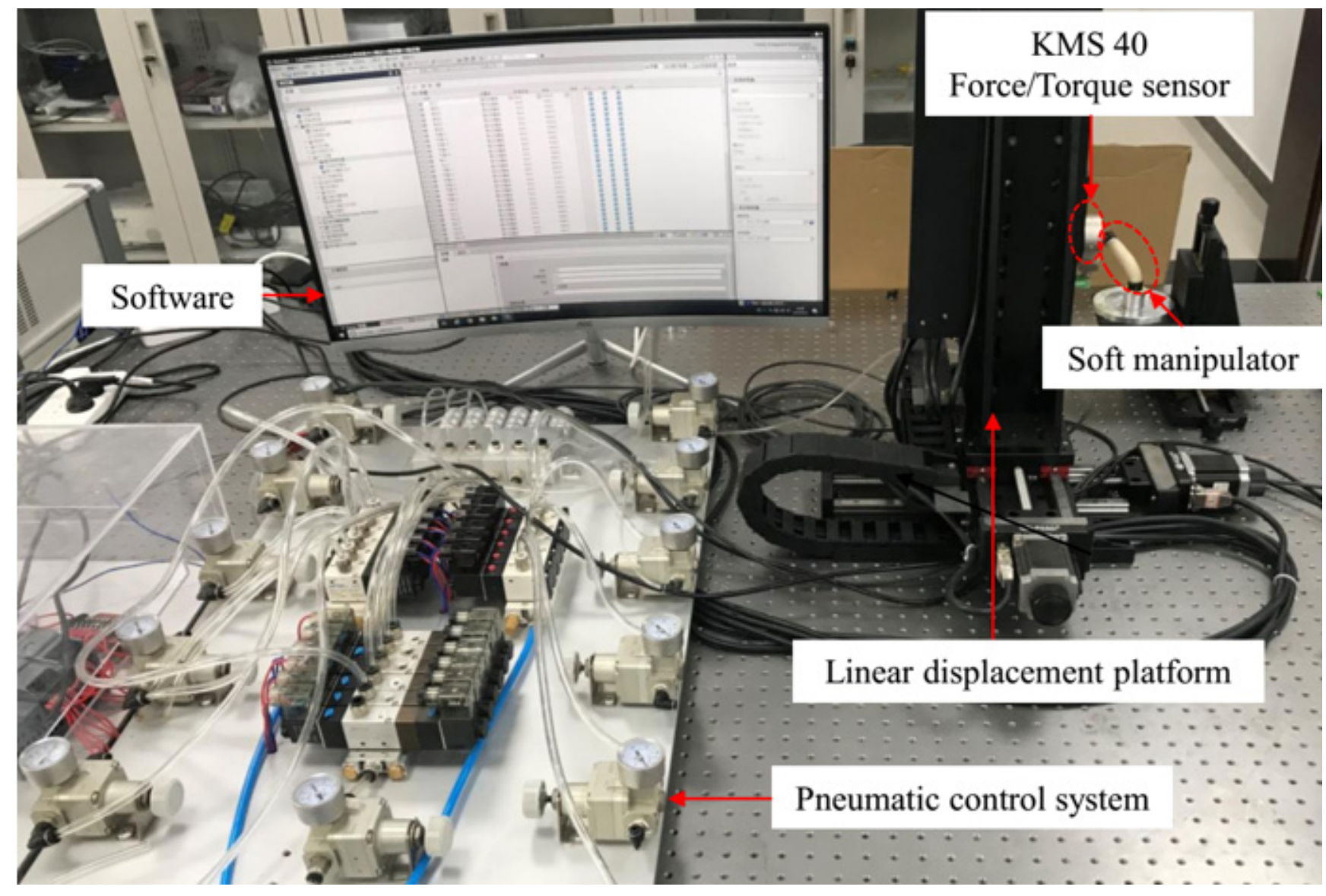

Figure 3. Experimental setup for evaluating the variable stiffness of the soft manipulator.

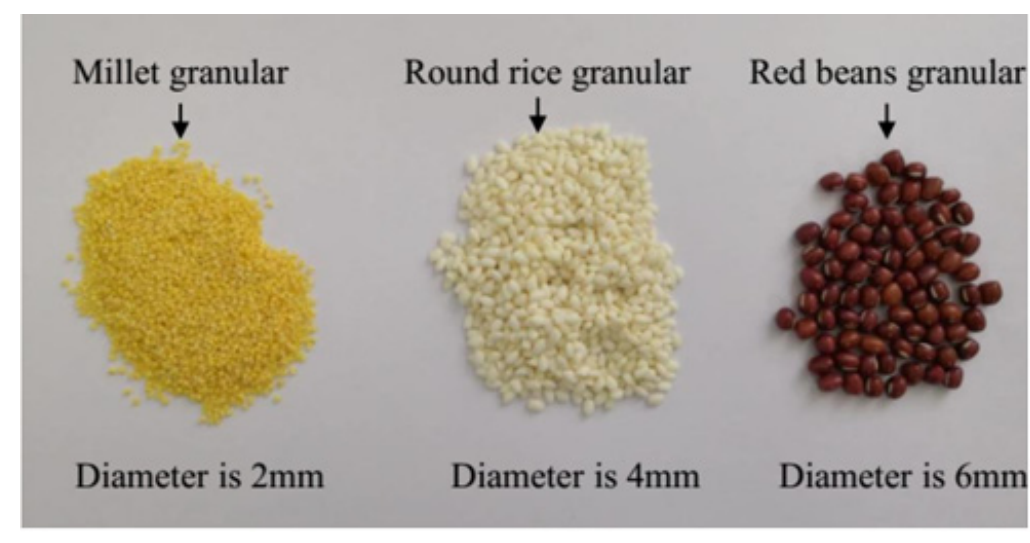

(a)

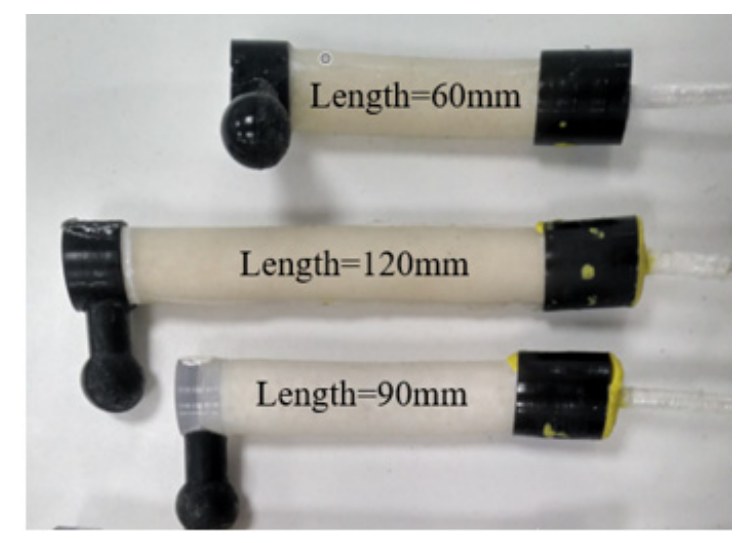

(b)

Figure 4. Prototypes of granules used in experiments and the soft silicone manipulator containing different granules. (a) Three granule types. (b) Soft silicone manipulators. 

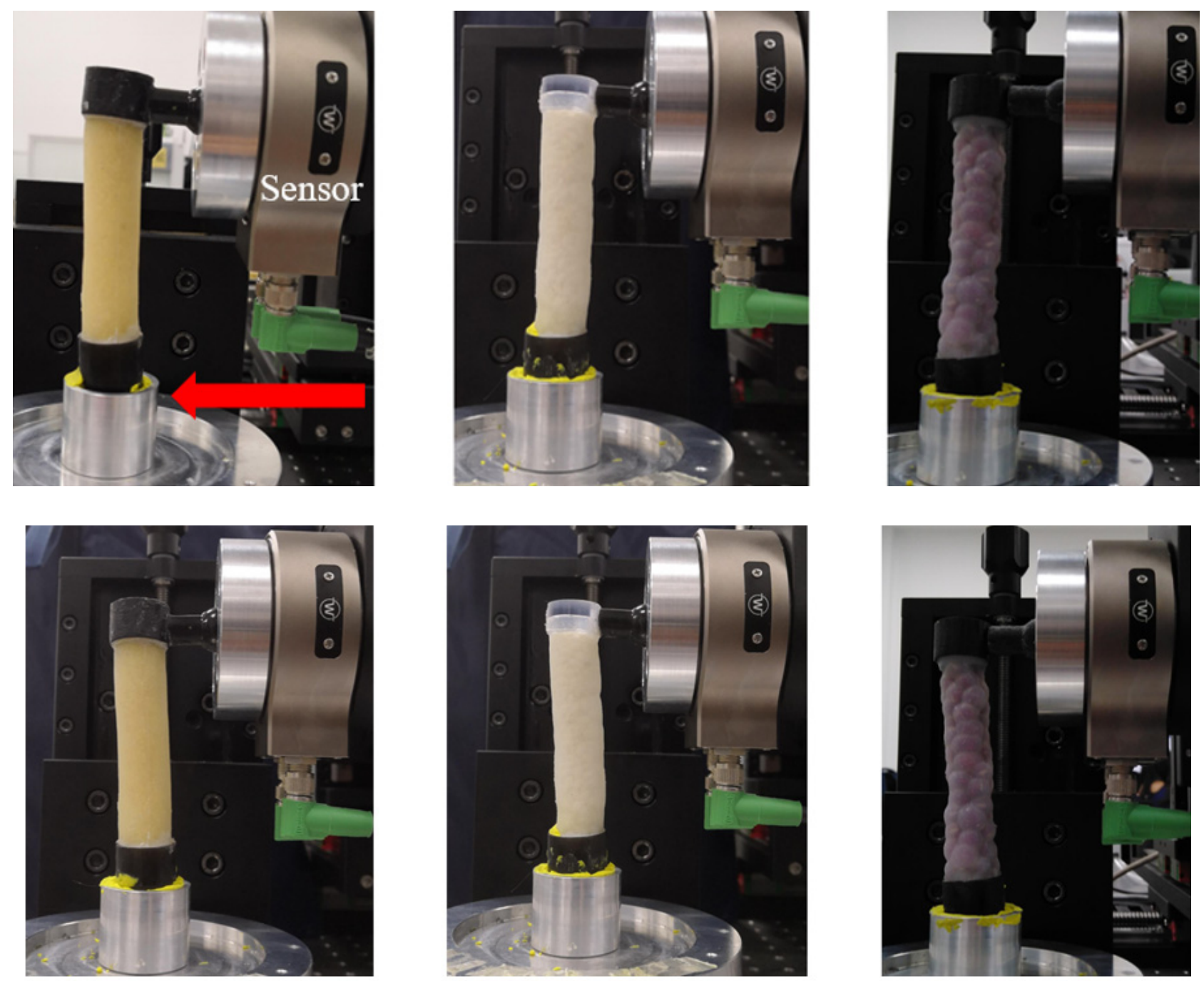

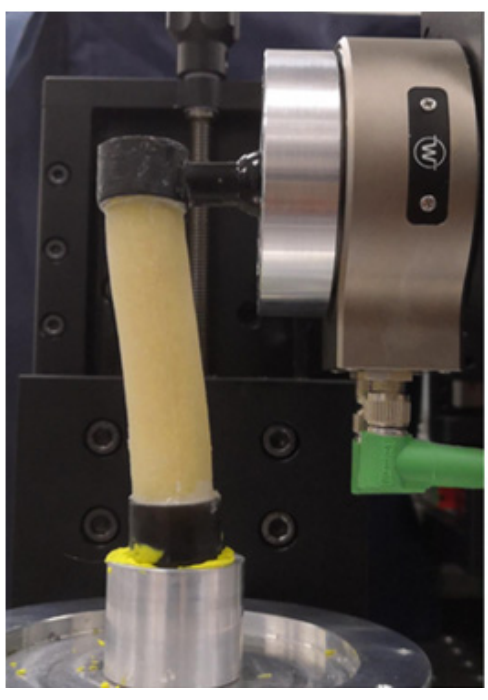

(a)

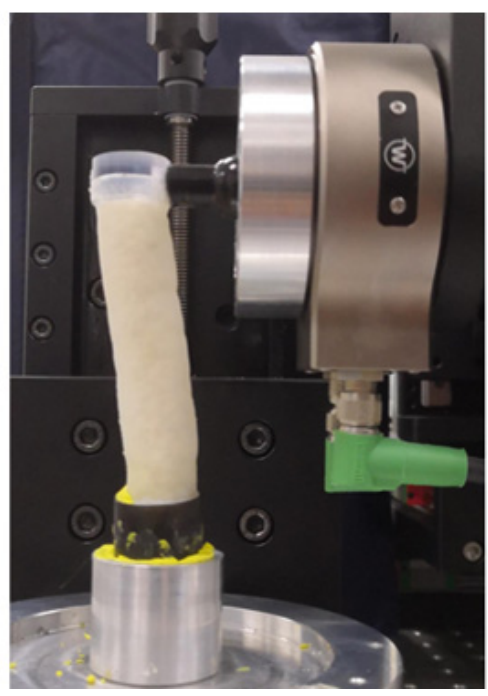

(b)

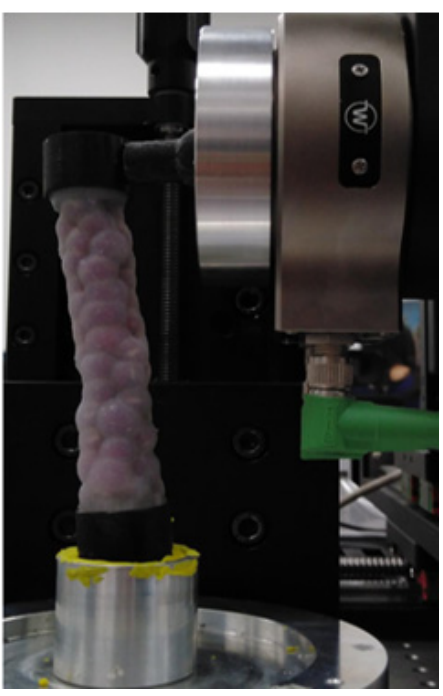

(c)

Figure 5. Experiments on the soft manipulator containing (a) millet, (b) round rice, and (c) red beans for the displacement platform movements of $0 \mathrm{~mm}$ (top), $5 \mathrm{~mm}$ (middle), and $10 \mathrm{~mm}$ (bottom). 


\section{Results and Discussion}

\subsection{Effect of Granular Size}

Figure $5 \mathrm{a}-\mathrm{c}$ shows the state of the soft manipulator containing three granules of different sizes, as the displacement platform was moved by 0,5 , and $10 \mathrm{~mm}$, respectively. The red arrow indicates the pushing direction of the displacement platform. Figure 6 shows the force of the soft manipulator (length $=60 \mathrm{~mm}$ ) with different types of granule for an input pressure of $80 \mathrm{kPa}$. When the soft manipulator was packed with millet, round rice, and red bean, the average force was $2.19,1.62$, and $0.47 \mathrm{~N}$, respectively. Thus, at a fixed manipulator length, input pressure, and pushing distance of the displacement platform, smaller granules result in greater stiffness force for the manipulator.

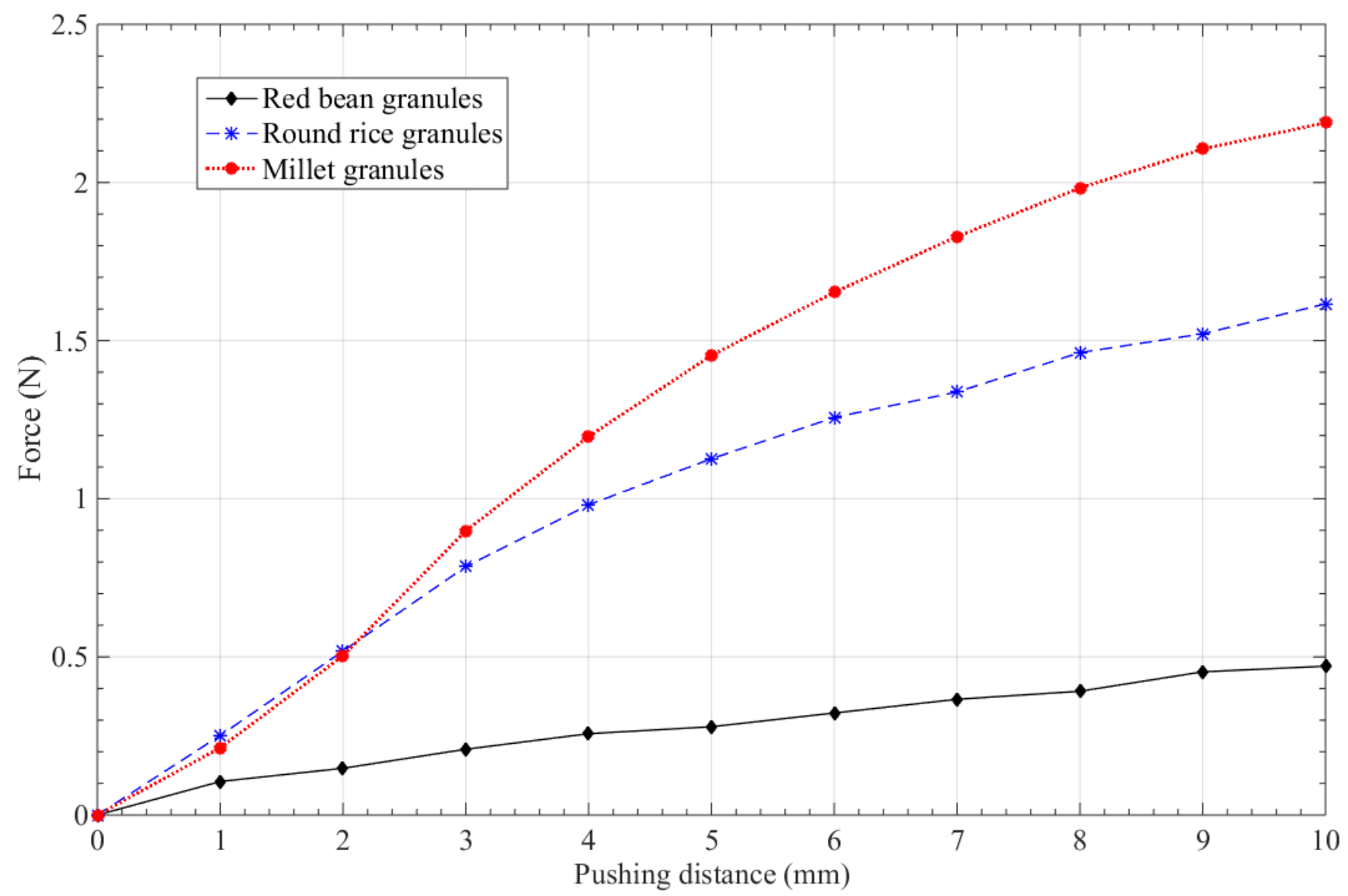

Figure 6. Experimental results of stiffness force for soft manipulators containing different types of granule.

In addition, to demonstrate the stability and consistency of the soft manipulator packed with different types of granules, four groups of experiments were conducted with three types of granules and different pushing distances (with the manipulator length and input pressure fixed). In this study, the deviation index $\left(D_{I}\right)$ is defined as the stability of the variable-stiffness performance of the soft manipulator, expressed as:

$$
D_{I}=\Delta f_{E} / \Delta f_{w} * 100 \%
$$

where $\Delta f_{E}$ represents the difference between each experimental result and the average value of force, and $\Delta f_{W}$ represents the sum of the forces at different displacements for each experiment. The average fluctuations in the stability of the soft manipulator containing millet, round rice, and red beans were $3.03 \%, 5.56 \%$, and $6.71 \%$, respectively (Figure 7). The maximum fluctuation between different experiments was less than $7 \%$; therefore, the variable-stiffness performance of the soft manipulator exhibited good stability and consistency with different granular sizes, especially for smaller granules. 


\subsection{Effect of Manipulator Length}

Figure 8 shows the state of the soft manipulator for different manipulator lengths and different displacement platform movements. Figure 9 shows the force of the soft manipulator (containing millet) with different lengths for an input pressure of $80 \mathrm{kPa}$. When the pushing distance of the displacement platform was fixed, the soft manipulator measuring $60 \mathrm{~mm}$ exhibited the largest force $(2.41 \mathrm{~N})$, whereas the one measuring $120 \mathrm{~mm}$ exhibited the smallest force $(1.42 \mathrm{~N})$. Therefore, at a fixed granular size, input pressure, and pushing distance of the displacement platform, a shorter manipulator length results in a greater stiffness force for the manipulator. Figure 10 shows the DI values for different manipulator lengths. For manipulator lengths of 60,90 , and $120 \mathrm{~mm}$, the DI values were $1.39 \%, 4.35 \%$, and $5.91 \%$, respectively. Clearly, the fluctuations are within $6 \%$, indicating good stability and consistency of the variable-stiffness performance of the soft manipulator at different lengths.

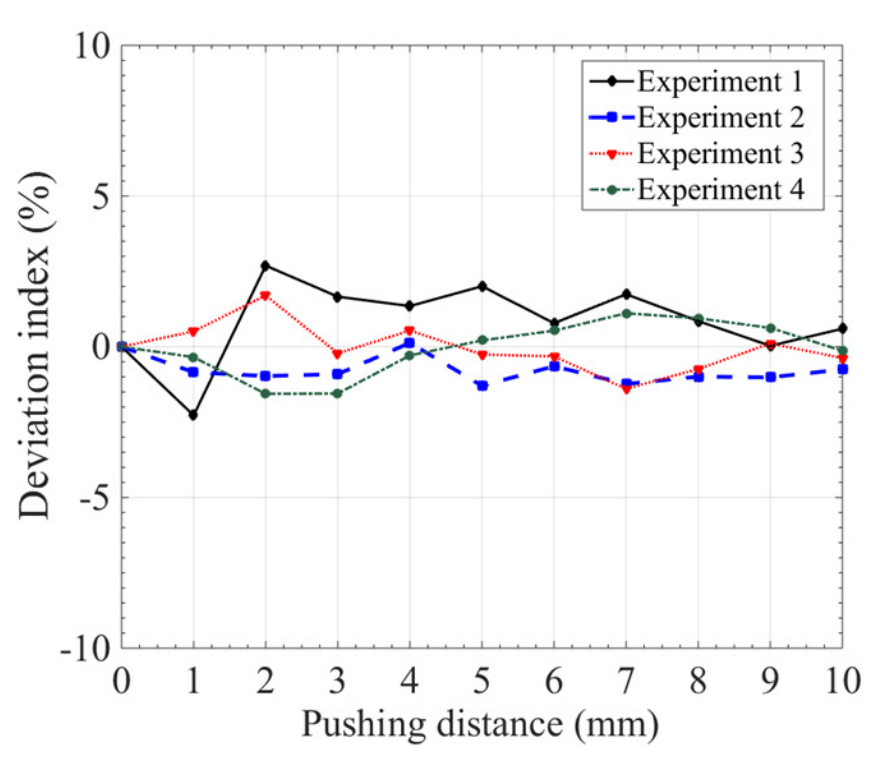

(a)

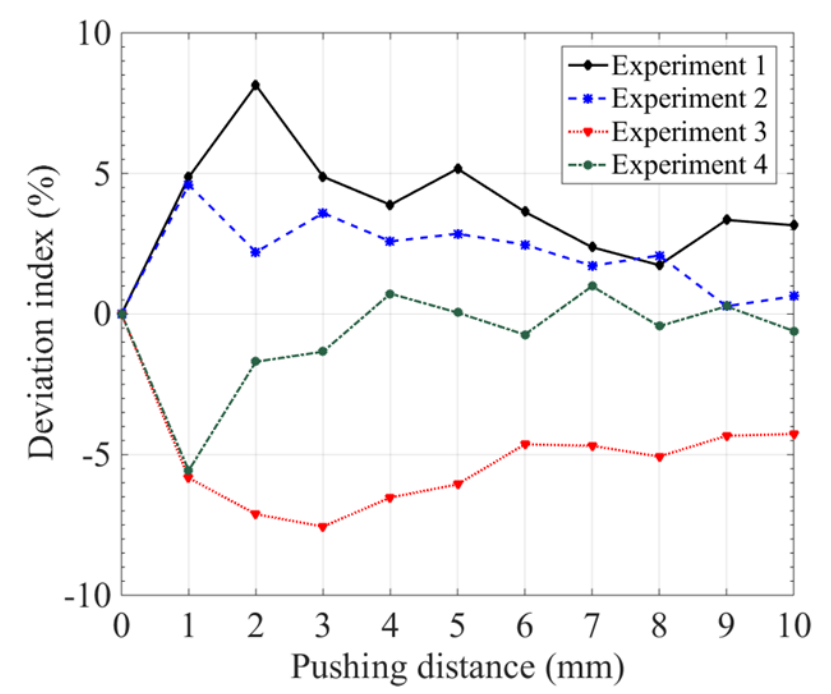

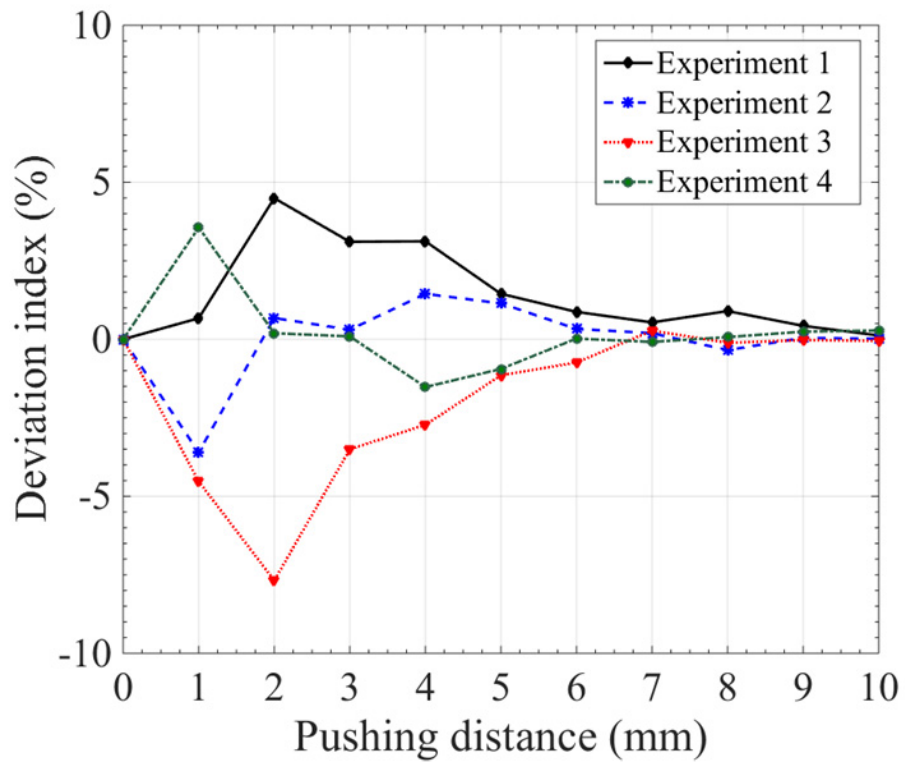

(b)

(c)

Figure 7. Stability comparison for soft manipulators containing (a) millet, (b) round rice, and (c) red beans. 


\subsection{Effect of Input Pressure}

Figure 11 shows the state of the soft manipulator under different pressures and different displacement platform movements. Figure 12 shows the force of the soft manipulator under different pressures. When the pushing distance of the displacement platform is fixed, a larger input pressure applied to the manipulator results in a larger force. The maximum force of the soft manipulator under input pressures of 44,56, 68, 80, and $92 \mathrm{kPa}$ is $1.45,1.65,1.96,2.19$, and $2.36 \mathrm{~N}$, respectively. For the same input pressures, the maximum DI values are $3.71 \%, 4.24 \%, 2.56 \%, 5.70 \%$, and $2.57 \%$, respectively, indicating that the variable-stiffness performance of the soft manipulator has good stability and consistency at different input pressures.
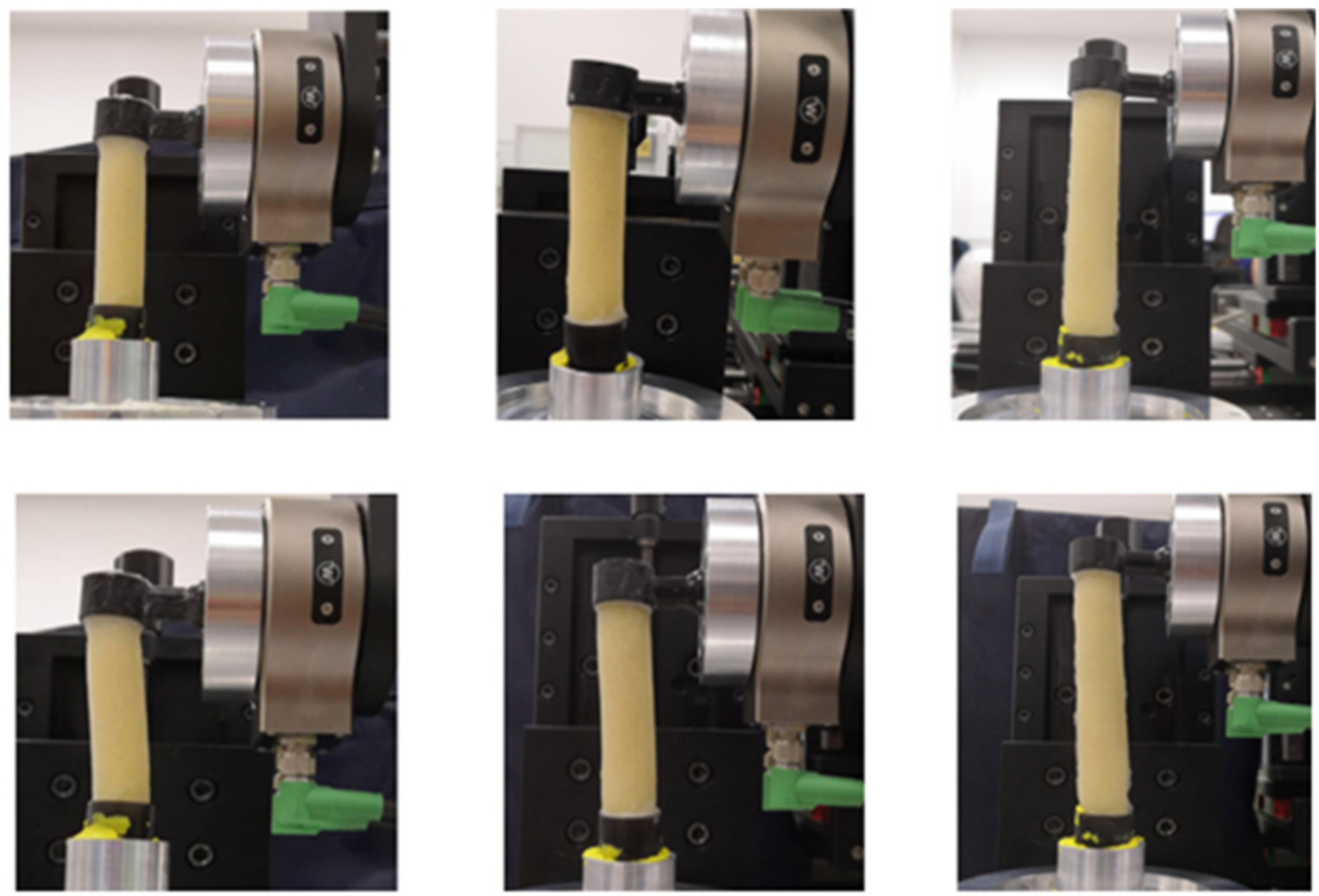

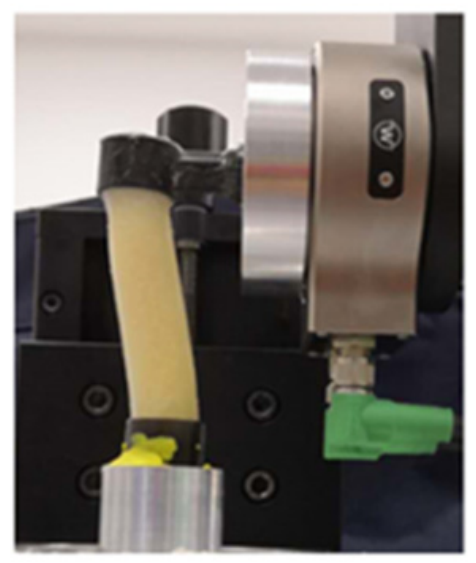

(a)

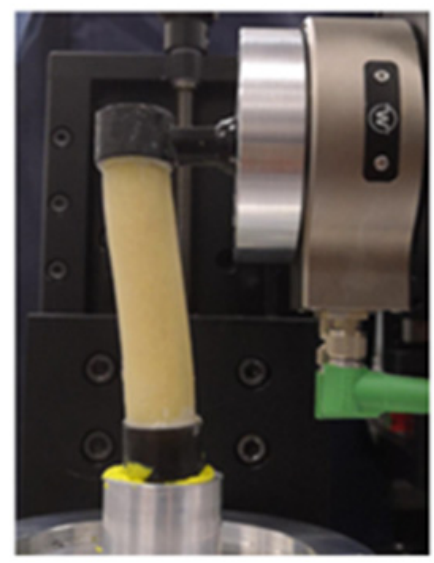

(b)

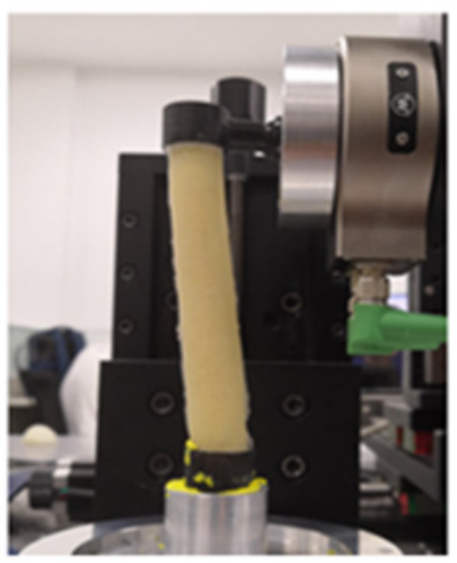

(c)

Figure 8. Experiments on the soft manipulator measuring (a) 60, (b) 90, and (c) $120 \mathrm{~mm}$ for displacement platform movements of $0 \mathrm{~mm}$ (top), $5 \mathrm{~mm}$ (middle), and $10 \mathrm{~mm}$ (bottom). 


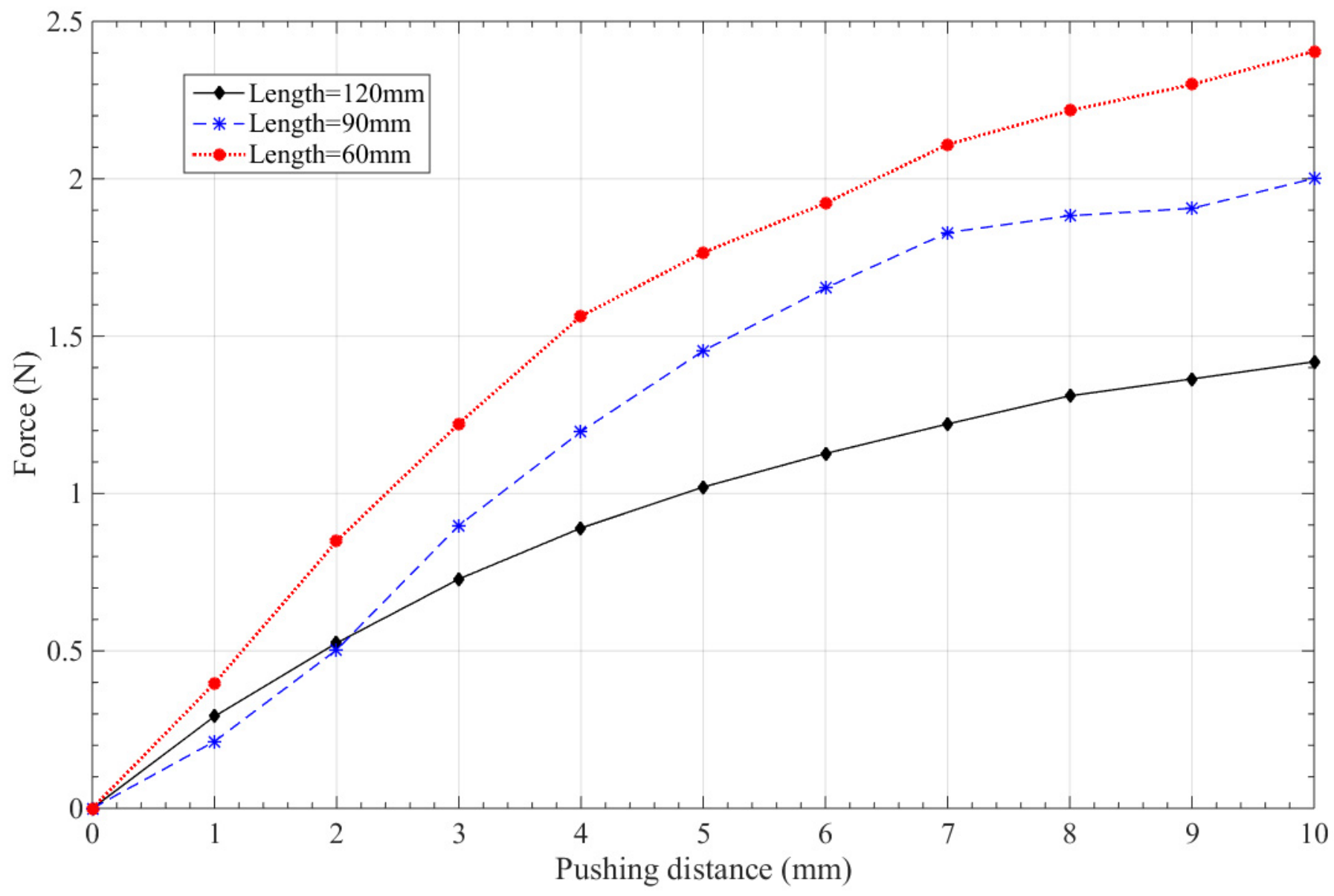

Figure 9. Experimental results of pushing distance and stiffness force for soft manipulators of different lengths.

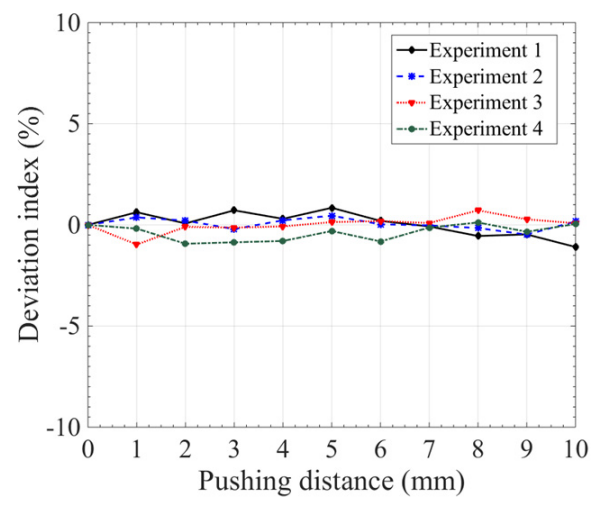

(a)

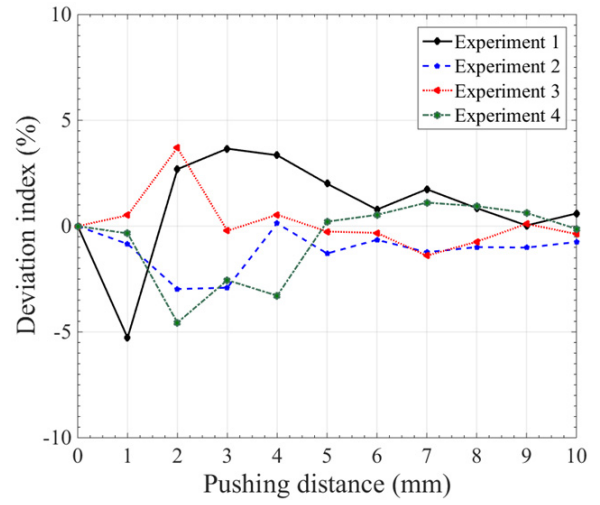

(b)

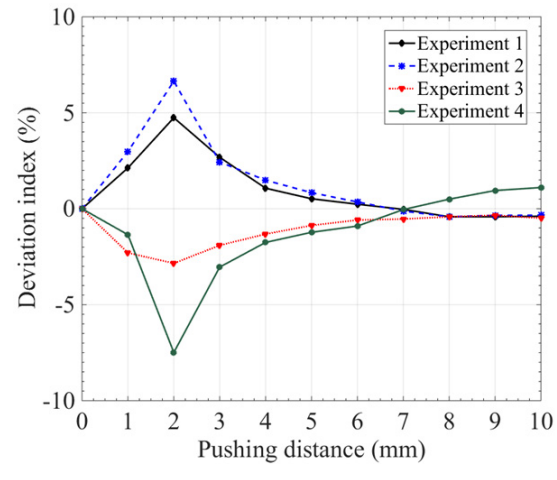

(c)

Figure 10. Stability comparison for soft manipulators measuring (a) 60, (b) 90, and (c) $120 \mathrm{~mm}$.

Figure 13 shows the experiment fluctuations for assessment of the effects of input pressure. For input pressures of 44,56, 68, 80, and $92 \mathrm{kPa}$, the maximum fluctuating intervals of the experiment were $3.71 \%, 4.24 \%, 2.56 \%, 5.70 \%$, and $2.57 \%$, respectively, indicating that the variable-stiffness performance of the soft manipulator has good stability and consistency. 

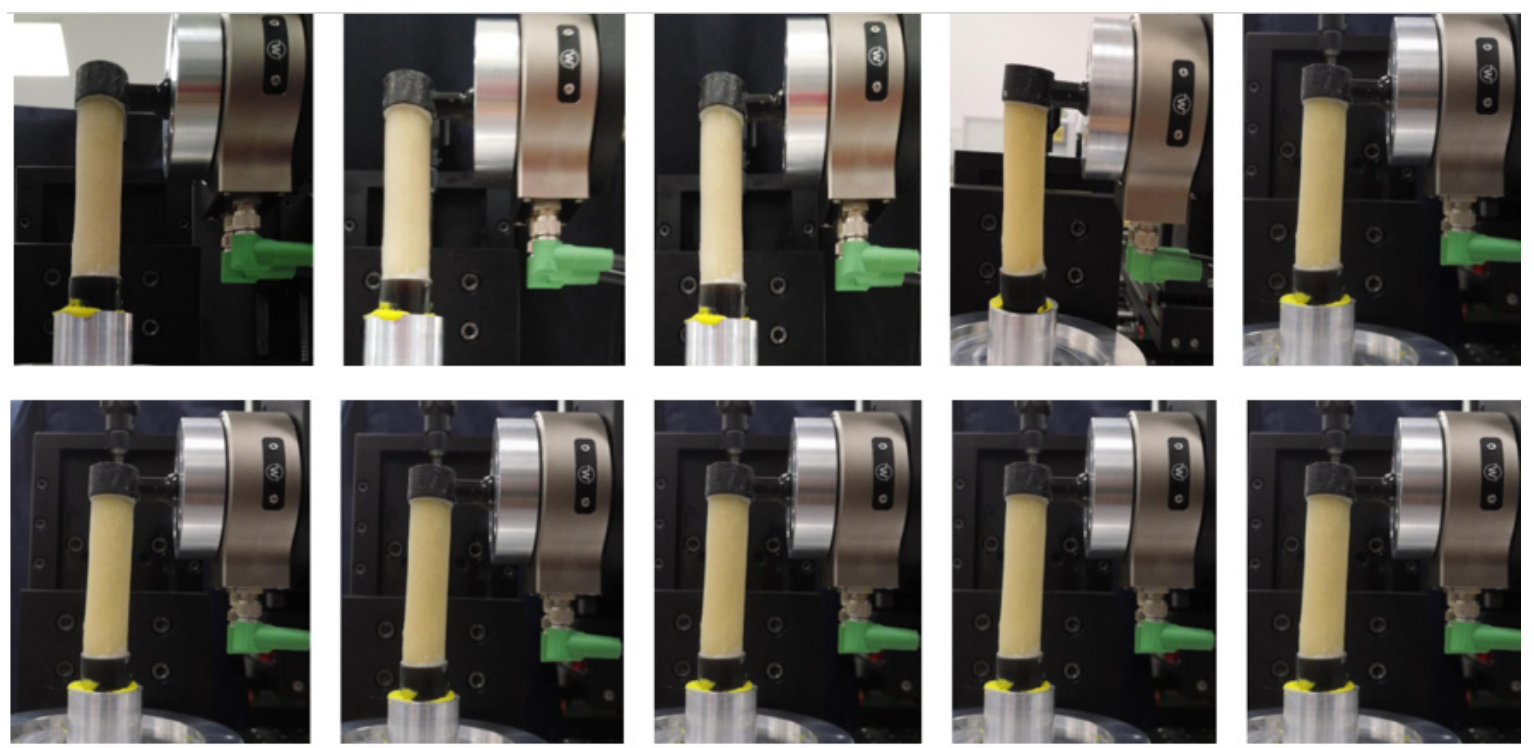

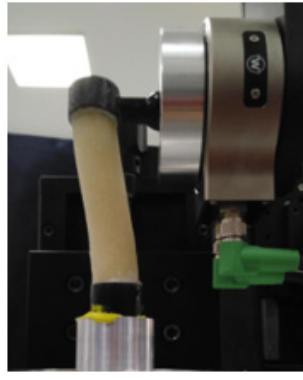

(a)

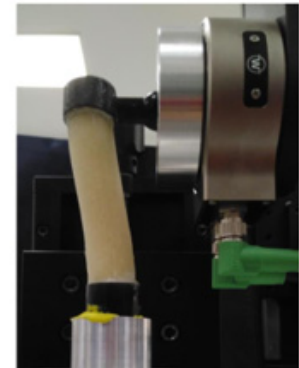

(b)

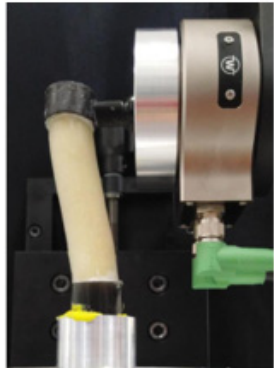

(c)

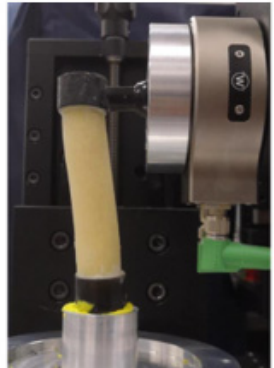

(d)

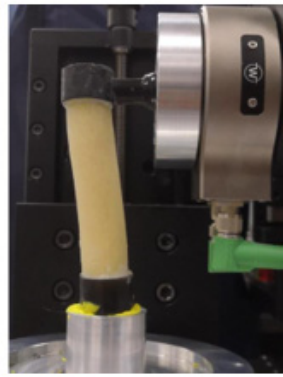

(e)

Figure 11. Experiments on the soft manipulator under input pressures of: (a) 44, (b) 56, (c) 68, (d) 80, and (e) $92 \mathrm{kPa}$ for displacement platform movements of $0 \mathrm{~mm}$ (top), $5 \mathrm{~mm}$ (middle), and $10 \mathrm{~mm}$ (bottom).

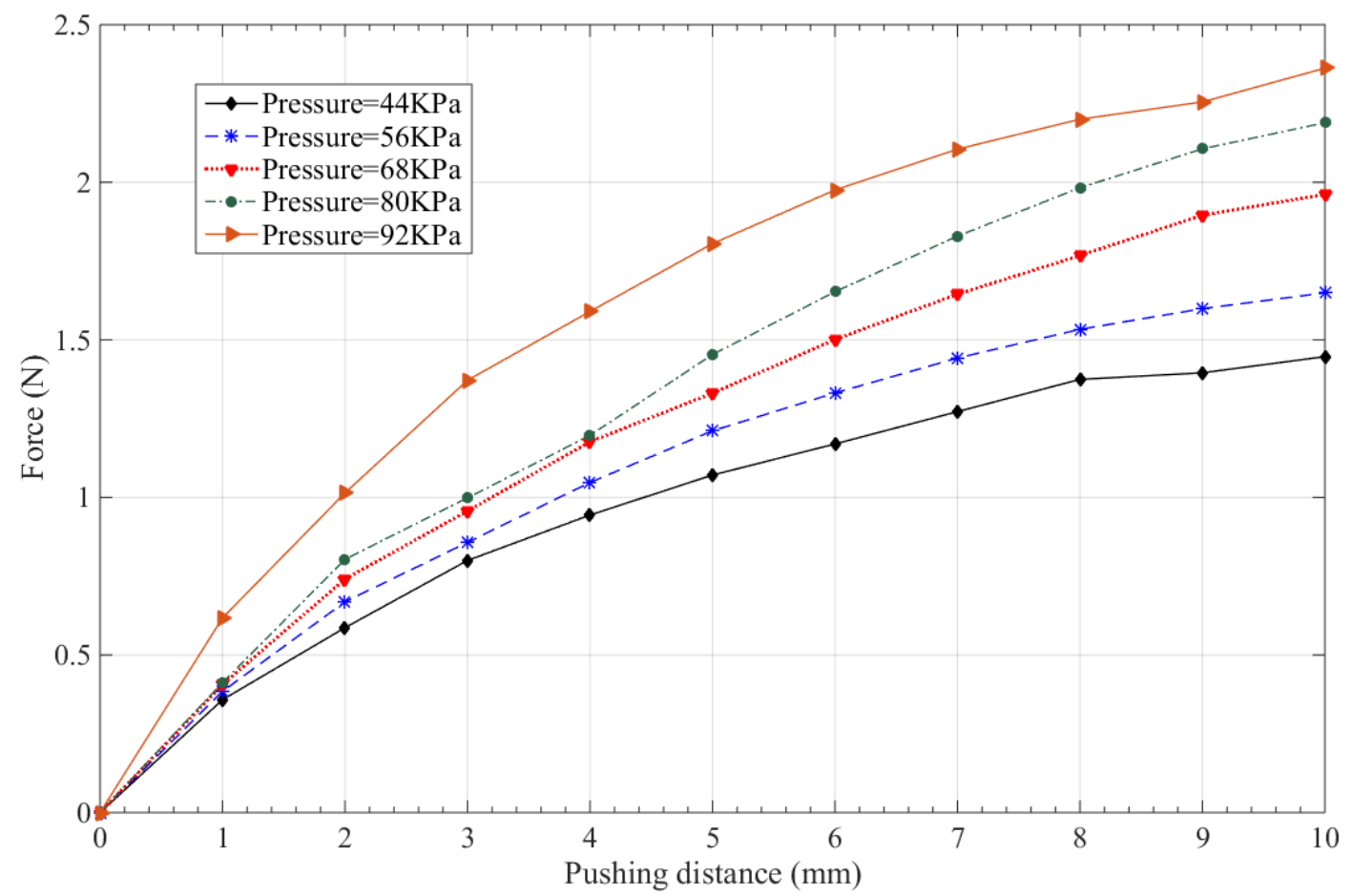

Figure 12. Experimental results of pushing distance and stiffness force for the soft manipulator under different input pressures. 


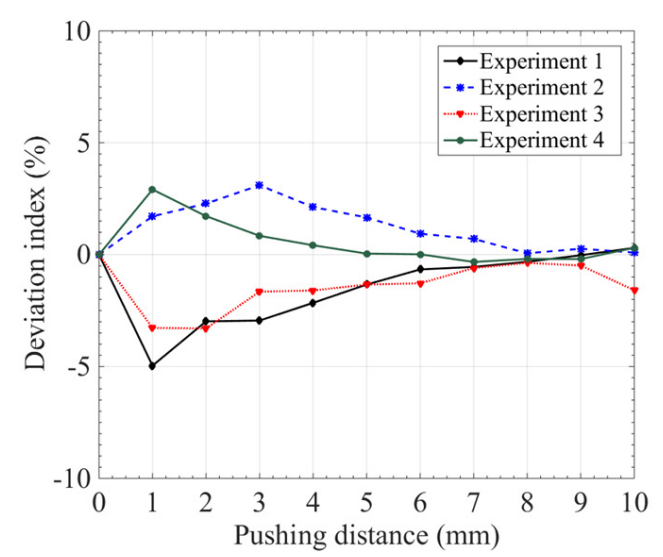

(a)

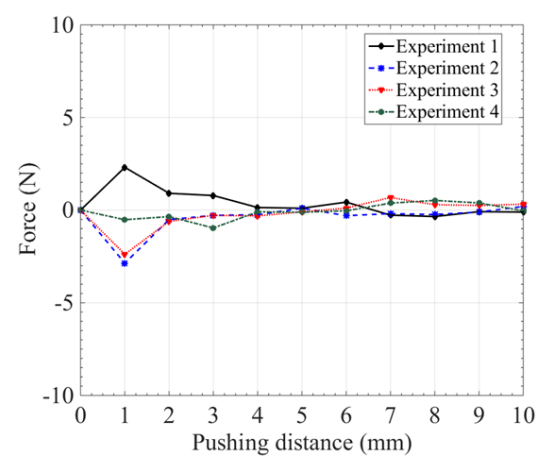

(c)

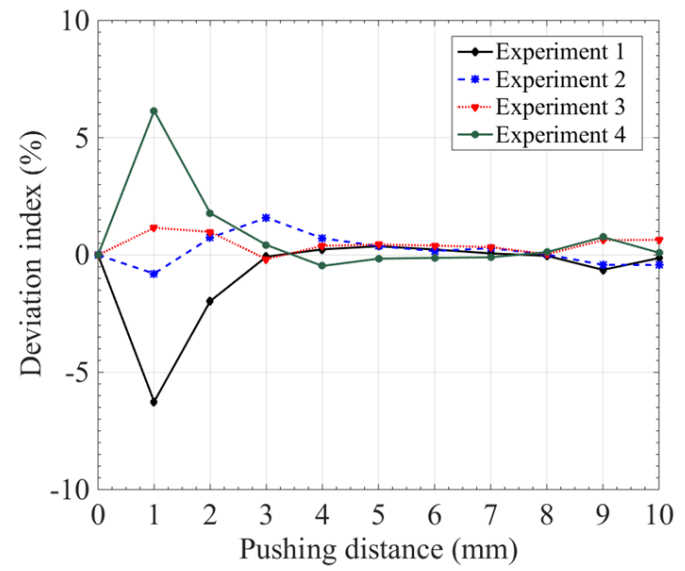

(b)

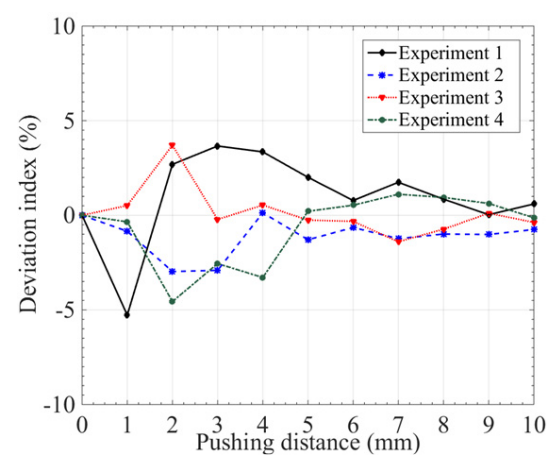

(d)

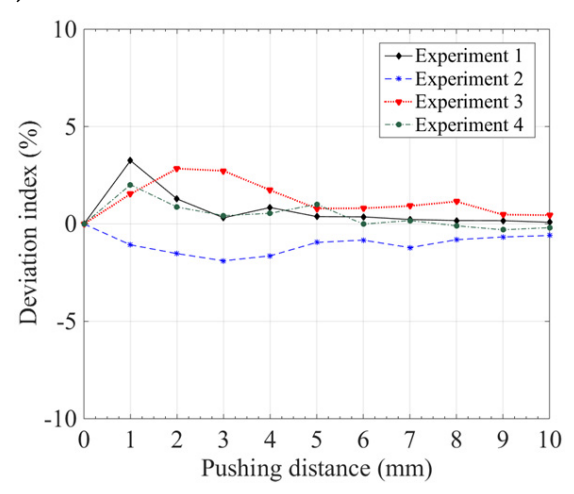

(e)

Figure 13. Stability comparison for the soft manipulator under different pressures: (a) 44, (b) 56, (c) 68, (d) 80, and (e) $92 \mathrm{kPa}$.

The above experimental results indicate that smaller granules, shorter manipulator lengths, and larger input pressures result in larger stiffness forces of the soft manipulator. The maximum stiffness force of the soft manipulator was found to be $2.41 \mathrm{~N}$. Therefore, to meet the practical application requirements of cardiac fixation during an OPCAB surgery, the flexible arm of the soft cardiac fixator can be designed with a length of $60 \mathrm{~mm}$ and packed with millets, and its variable-stiffness performance can be achieved through different input pressure controls. Nevertheless, this study possesses limitations in that no experiment was conducted to verify the variable stiffness of the flexible arm for practical applications; moreover, the model of the flexible arm was simplified in this work. Our future work will focus on more accurate finite element models with careful considerations regarding contact and friction.

\section{Conclusions}

In this study, a soft cardiac fixator with a variable-stiffness flexible arm that can be used for cardiac fixation during OPCAB surgery was developed. To evaluate the variable stiffness of the flexible arm, three simplified soft silicone manipulators with lengths of 60,90 , and $120 \mathrm{~mm}$ were fabricated. These manipulators can actuate, soften, or stiffen independently along the length of the arm by combining granular jamming with input pressure. The experimental results demonstrated that the variable-stiffness performance of the soft manipulator is influenced by its length, the size of the granules, and the input pressure. The force of the soft manipulator can reach $2.41 \mathrm{~N}$, which meets the practical application requirements for cardiac fixation during OPCAB surgery. Moreover, the maximum force variability between different experiments was less than $7 \%$, indicating 
that the variable-stiffness performance of the soft manipulator has good stability and consistency. However, the length setting of the flexible arm was limited in this study, and other potential safety issues such as air leakage in clinical applications may exist. Therefore, the size of future flexible arm studies will be appropriately expanded, the overall system integration of the fixator will be completed, and performance studies will be conducted in a simulated surgical environment to improve the safety of this system. The dynamic behaviour and closed-loop control of the soft cardiac fixator will also be investigated.

Author Contributions: L.G. is the main author of this paper. She proposed the main idea and plan, designed the experiment, and wrote the manuscript. Y.H. analysed the proposed method. L.Z. suggested improvements. G.S. suggested improvements to the main ideas regarding the research and writing. H.Z. revised the paper for language. All authors have read and agreed to the published version of the manuscript.

Funding: This work was supported by the National Natural Science Foundation of China (61903041). This work was also supported by the Natural Science Foundation of Beijing, China (4204101, 7202017), the Research Project of Beijing Education Committee (KM201911232005), and the Preferential Funding for Postdoctoral Research Projects of Hebei Province of China (B2020003020).

Institutional Review Board Statement: Not applicable.

Informed Consent Statement: Not applicable.

Data Availability Statement: The data used in this study are openly available in the public domain.

Conflicts of Interest: The authors declare no conflict of interest.

\section{References}

1. Soltoski, P.; Bergsland, J.; Salerno, T.A.; Karamanoukian, H.L.; D'Anconet, G.; Ricci, M.; Panosal, A.L. Techniques of Exposure and Stabilization in Off-Pump Coronary Artery Bypass Graft. Echocardiography 1999, 2, 392-400. [CrossRef]

2. Cianchetti, M.; Ranzani, T.; Gerboni, G.; De Falco, I.; Laschi, C.; Menciassi, A. STIFF-FLOP surgical manipulator: Mechanical design and experimental characterization of the single module. In Proceedings of the 2013 IEEE/RSJ International Conference on Intelligent Robots and Systems, Tokyo, Japan, 3-7 November 2013; pp. 3576-3581.

3. Nabil, E.; Belhassen-Chedli, B.; Grigore, G. Soft material modeling for robotic task formulation and controlling the muscle separation process. Robot. Comput. Integr. Manuf. 2015, 32, 37-52. [CrossRef]

4. Tang, G.; Webb, P.; Thrower, J. The development and evaluation of Robot Light Skin: A novel robot signalling system to improve communication in industrial human-robot collaboration. Robot. Comput. Integr. Manuf. 2019, 56, 85-94. [CrossRef]

5. Ranzani, T.; Gerboni, G.; Cianchetti, M.; Menciassi, A. A bioinspired soft manipulator for minimally invasive surgery. Bioinspir. Biomim. 2015, 10, 035008. [CrossRef]

6. Zhao, H.; Jalving, J.; Huang, R.; Knepper, R.; Ruina, A.; Shepherd, R. A Helping Hand: Soft Orthosis with Integrated Optical Strain Sensors and EMG Control. IEEE Robot. Autom. Mag. 2016, 23, 55-64. [CrossRef]

7. Polygerinos, P.; Wang, Z.; Galloway, K.C. Soft manipulatoric glove for combined assistance and at-home rehabilitation. Manip. Auton. Syst. 2015, 73, 135-143. [CrossRef]

8. Sareh, S.; Althoefer, K.; Li, M.; Noh, Y.; Tramacere, F.; Sareh, P.; Mazzolai, B.; Kovac, M. Anchoring like octopus: Biologically inspired soft artificial sucker. J. R. Soc. Interface 2017, 14, 20170395. [CrossRef]

9. Zolfagharian, A.; Kouzani, A.Z.; Khoo, S.Y.; Moghadam, A.A.A.; Gibson, I.; Kaynak, A. Evolution of 3D printed soft actuators. Sens. Actuators A Phys. 2016, 250, 258-272. [CrossRef]

10. Laschi, C.; Mazzolai, B.; Cianchetti, M. Soft robotics: Technologies and systems pushing the boundaries of robot abilities. Sci. Robot. 2016, 1, eaah3690. [CrossRef]

11. Luo, M.; Skorina, E.H.; Tao, W.; Chen, F.; Ozel, S.; Sun, Y.; Onal, C.D. Toward Modular Soft Robotics: Proprioceptive Curvature Sensing and Sliding-Mode Control of Soft Bidirectional Bending Modules. Soft Robot. 2017, 4, 117-125. [CrossRef]

12. Robertson, M.A.; Paik, J. New soft robots really suck: Vacuum-powered systems empower diverse capabilities. Sci. Robot. 2017, 2, eaan6357. [CrossRef]

13. Sandesh, R.S.; Venkatesan, N. Novel approach to control of robotic hand using flex sensors. Int. J. Robot. Autom. 2014, 3, 3234-3244.

14. Ranzani, T.; Cianchetti, M.; Gerboni, G.; De Falco, I.; Menciassi, A. A Soft Modular Manipulator for Minimally Invasive Surgery: Design and Characterization of a Single Module. IEEE Trans. Robot. 2016, 32, 187-200. [CrossRef]

15. Zouari, L.; Abid, H.; Abid, M. Backstepping Controller for Electrically Driven Flexible Joint Manipulator Under Uncertainties. IAES Int. J. Robot. Autom. 2014, 4, 156-163. [CrossRef] 
16. Payne, C.J.; Wamala, I.; Bautista-Salinas, D.; Saeed, M.; Van Story, D.; Thalhofer, T.; Horvath, M.A.; Abah, C.; del Nido, P.J.; Walsh, C.J.; et al. Soft robotic ventricular assist device with septal bracing for therapy of heart failure. Sci. Robot. 2017, 2, eaan6736. [CrossRef]

17. Cheng, N.G.; Lobovsky, M.B.; Keating, S.J.; Setapen, A.M.; Gero, K.I.; Hosoi, A.E.; Iagnemma, K.D. Design and Analysis of a Robust, Low-cost, Highly Articulated manipulator enabled by jamming of granular media. In Proceedings of the 2012 IEEE International Conference on Robotics and Automation, St. Paul, MN, USA, 14-19 May 2012; pp. 4328-4333.

18. Kim, Y.-J.; Cheng, S.; Kim, S.; Iagnemma, K. A Novel Layer Jamming Mechanism with Tunable Stiffness Capability for Minimally Invasive Surgery. IEEE Trans. Robot. 2013, 29, 1031-1042. [CrossRef]

19. Kim, Y.J.; Cheng, S.; Kim, S.; Iagnemma, K. A stiffness-adjustable hyper redundant manipulator using a variable neutral-line mechanism for minimally invasive surgery. IEEE Trans. Robot. 2014, 30, 382-395. [CrossRef]

20. Camarillo, D.B.; Milne, C.F.; Carlson, C.R.; Zinn, M.R.; Salisbury, J.K. Mechanics Modeling of Tendon-Driven Continuum Manipulators. IEEE Trans. Robot. 2008, 24, 1262-1273. [CrossRef]

21. Khodambashi, R.; Alsaid, Y.; Rico, R.; Marvi, H.; Peet, M.M.; Fisher, R.E.; Berman, S.; He, X.; Aukes, D.M. Heterogeneous Hydrogel Structures with Spatiotemporal Reconfigurability using Addressable and Tunable Voxels. Adv. Mater. 2021, 33 , 2005906. [CrossRef]

22. Park, J.E.; Jeon, J.; Park, S.J.; Won, S.; Ku, Z.; Wie, J.J. Enhancement of Magneto-Mechanical Actuation of Micropillar Arrays by Anisotropic Stress Distribution. Small 2020, 16, 2003179. [CrossRef] [PubMed]

23. Degani, A.; Choset, H.; Zubiate, B.; Ota, T.; Zenati, M. Highly articulated robotic probe for minimally invasive surgery. In Proceedings of the 30th Annual International Conference of the IEEE Engineering in Medicine and Biology Society, Vancouver, BC, Canada, 17 June 2008; pp. 3273-3276. [CrossRef]

24. Simaan, N. Snake-Like Units Using Flexible Backbones and Actuation Redundancy for Enhanced Miniaturization. In Proceedings of the 2005 IEEE International Conference on Robotics and Automation, Barcelona, Spain, 18-22 April 2005; pp. 3012-3017.

25. Jiang, S.; Chen, B.; Qi, F.; Cao, Y.; Ju, F.; Bai, D.; Wang, Y. A variable-stiffness continuum manipulators by an SMA-based sheath in minimally invasive surgery. Int. J. Med Robot. Comput. Assist. Surg. 2020, 16, e2081. [CrossRef]

26. Dupont, P.E.; Lock, J.; Itkowitz, B.; Butler, E.J. Design and Control of Concentric-Tube Robots. IEEE Trans. Robot. 2010, 26, 209-225. [CrossRef]

27. Suresh, A.; Udupa, G.; Gaba, D. Design and development of soft robotic hand for vertical farming in spacecraft. IAES Int. J. Robot. Autom. 2020, 9, 6-16. [CrossRef]

28. Cha, H.-J.; Koh, K.C.; Yi, B.-J. Stiffness modeling of a soft finger. Int. J. Control. Autom. Syst. 2014, 12, 111-117. [CrossRef]

29. Stilli, A.; Wurdemann, H.A.; Althoefer, K. A Novel Concept for Safe, Stiffness-Controllable Robot Links. Soft Robot. 2017, 4, 16-22. [CrossRef] [PubMed]

30. Morrison, T.; Su, H.-J. Stiffness modeling of a variable stiffness compliant link. Mech. Mach. Theory 2020, 153, 104021. [CrossRef]

31. Yufei, H.; Tianmiao, W.; Xi, F.; Kang, Y.; Ling, M.; Juan, G.; Li, W. A variable stiffness soft robotic gripper with low-melting-point alloy. In Proceedings of the 2017 36th Chinese Control Conference, Dalian, China, 26-28 July 2017; pp. 6781-6786.

32. Wang, H.; Chen, Z.; Zuo, S. Flexible Manipulator with Low-Melting-Point Alloy Actuation and Variable Stiffness. Soft Robot. 2021. [CrossRef]

33. Teall, O.; Pilegis, M.; Sweeney, J.; Gough, T.; Thompson, G.; Jefferson, A.; Lark, R.; Gardner, D. Development of high shrinkage polyethylene terephthalate (PET) shape memory polymer tendons for concrete crack closure. Smart Mater. Struct. 2017, $26,045006$. [CrossRef]

34. Mitsuda, T.; Kuge, S.; Wakabayashi, M.; Kawamura, S. Wearable haptic display by the use of a Particle Mechanical Constraint. In Proceedings of the Proceedings 10th Symposium on Haptic Interfaces for Virtual Environment and Teleoperator Systems, Orlando, FL, USA, 24-25 March 2002; pp. 153-158.

35. Amend, J.R.; Brown, E.; Rodenberg, N.; Jaeger, H.M.; Lipson, H. A Positive Pressure Universal Gripper Based on the Jamming of Granular Material. IEEE Trans. Robot. 2012, 28, 341-350. [CrossRef]

36. Jiang, A.; Xynogalas, G.; Dasgupta, P.; Althoefer, K.; Nanayakkara, T. Design of a variable stiffness flexible manipulator with composite granular jamming and membrane coupling. In Proceedings of the 2012 IEEE/RSJ International Conference on Intelligent Robots and Systems, Vilamoura-Algarve, Portugal, 7-12 October 2012; pp. 2922-2927.

37. Tomokazu, T.; Kikuchi, S.; Suzuki, M.; Aoyagi, S. Vacuum gripper imitated octopus sucker-effect of liquid membrane for absorption. In Proceedings of the 2015 IEEE/RSJ International Conference on Intelligent Robots and Systems, Hamburg, Germany, 28 September-3 October 2015; pp. 2929-2936. 